\title{
An Object Interface for Interoperability of Image Processing Parallel Library in a Distributed Environment
}

\author{
Andrea Clematis, Daniele D'Agostino, and Antonella Galizia \\ IMATI-CNR, Via de Marini 6, 16149 Genova, Italy \\ \{antonella, clematis, dago\}@ge.imati.cnr.it
}

\begin{abstract}
Image processing applications are computing demanding and since a long time much attention has been paid to the use of parallel processing. Emerging distributed and Grid based architectures represent new and well suited platforms that promise the availability of the required computational power. In this direction image processing has to evolve to heterogeneous environments, and a crucial aspect is represented by the interoperability and reuse of available and high performance code. This paper describes our experience in the development of PIMA(GE) ${ }^{2}$, Parallel IMAGE processing GEnoa server, obtained wrapping a library using the CORBA framework. Our aim is to obtain a high level of flexibility and dynamicity in the server architecture with a possible limited overhead. The design of a hierarchy of image processing operation objects and the development of the server interface are discussed.
\end{abstract}

\section{Introduction}

During the last decades Image Processing has become a topic of interest for a broad scientific community, since the emerging digital technologies allow to process complex signals; on the other hand image processing growth is empowered by its applications, covering several fields of interest such as medicine, industries, military, meteorology, and earth observation. The variety of problems arising with the different application domains leads to the evolution of several specific analysis and processing techniques, that mainly differ in the type of images they are related with, or in the task they should perform. However capturing, processing, and managing images require an enormous computational effort, often too high for a single processor architecture. The demand for computing resources is increasing and distributed computing environments provide an effective and attractive solution. The emerging technologies to overcome these requirements lead to distributed architectures and Computational Grids [8].

To satisfy image processing requirements, it is important to explore the use of Computational Grids to allow the resolution of computing intensive applications and at the same time preserving previously developed software. In this direction a great interest is dedicated to interoperability and reuse of legacy code. In the recent past different technologies and methodologies have been developed to enable this process. The generally adopted solution is the legacy code 
encapsulation: the code is left in its environment and dynamically connected to the new technologies through a wrapper, allowing the software performs in a Client/Server system [20. In this way it is possible to profit of the advantages offered by the new infrastructures and to keep previously developed softwares, still representing a useful support to solve problems.

In the presented experience we encapsulate a parallel image processing library in a component framework, CORBA [3], in order to develop PIMA(GE) ${ }^{2}$, Parallel IMAGE processing GEnoa distributed and heterogeneous server. The applications developed using PIMA $(\mathrm{GE})^{2}$ may be integrated in a Grid architecture. In particular the server implements the most common image processing operations performed in a data parallel fashion; the parallelism is hidden from the users and totally managed by the server, that also applies a transparent optimization policy. The legacy code, i.e. the library, is totally reused.

The server also represents a way to combine Single Program Multiple Data, SPMD, parallel computing model implemented with MPI [13, and distributed programming technologies, using CORBA, without modifications to the Object Management Group [15, OMG standard.

In the paper the main emphasis is given to the definition of the PIMA $(\mathrm{GE})^{2}$ interface, that represents in our opinion, one of the most important elements in this kind of work, in order to simplify the reuse, ensuring flexibility and interoperability. A particular interesting aspect is that legacy code is a parallel image processing library. This is kept in due consideration during the interface planning and the architecture development.

The paper is organized as follow: in the next Sect. the evolution of the library concept in a distributed and heterogeneous environment is discussed; in Sect. 3 the wrapping phase and the definition of the Application Programming Interface (API) of the server are described; in Sect. 4 the PIMA(GE) ${ }^{2}$ architecture is briefly detailed; we present experimental results and conclusions in Sect. 5 .

\section{Moving Towards Heterogeneous Dynamic Image Processing}

In order to allow the use of image processing tools in distributed and Grid environments, it is necessary to evolve from library to a component based server. We have to keep the good features of the library and add to them other important characteristics, such as interoperability, and performance portability, to permit the integration in distributed and heterogeneous environments. Our aim is to obtain a high level of flexibility and dynamicity in the server architecture with an acceptable overhead.

The parallel image processing library, used during this work, is a still ongoing project implemented in C++ and MPI 9]. The library implements the most common image processing operations, according to the classification provided in Image Algebra [18. The operations are performed in a data parallel fashion; the parallelism is hidden from the users and totally managed by the library. It also provides a performance optimization oriented to different levels: an optimization 
policy is applied to perform a suitable management of communication and memory operations; the data distribution is oriented to obtain load balancing; and others ongoing efforts are dedicated to the exploitation of memory hierarchies. Also the optimization aspects are transparent for the users.

A library is often considered as a static tool, designed to develop the user's own applications; it is mainly designed to be executed on an homogeneous architecture in order to develop static applications. However in the era of distributed and Grid computing, the concept of library is evolving. Moving the software towards distributed, dynamic and heterogeneous environments, we are transforming it in a component-based bundle that can be accessed using a server based interaction. It means that the functions performed by the library are evolved into components, and an application becomes a dynamic components concatenation, executed on an heterogeneous infrastructures. The server is no longer a static entity, it is able to manage multiple requests performed by different distributed clients.

Since the library is not modified and totally reused during the encapsulation process, the server inherits its features, such as transparence, high performance and completeness. These features should be improved by adding further properties, such as distributed and heterogeneous executions, software extensibility and interoperability, dynamicity and portability on the Grid. We obtain a dynamic interaction model that permits to accommodate request arriving from heterogeneous Client in a distributed environment.

\subsection{Related Works}

In the image processing domain it is possible to find different examples of parallel libraries, for example VSIPL++ [12, ParHorus [19, PIPT [17. They provide object-oriented image processing code, and ensure high performance executions; in fact they are improved with performance optimization policy and are compliant to the standard features ensured by a scientific library. There are also several on-going efforts aiming at the exploitation of distributed and heterogeneous environments reusing the previous developed software. Important and successful projects are represented by VGrADS [10, Cactus [2], NetSolve [14, that aim to the integration of numerical libraries in Grid environments. The need of migration to distributed and Grid environments is even present in the image processing community, as different and actual works show, 4, 1, 11.

\section{The Definition of the PIMA(GE) ${ }^{2}$ Interface}

To allow a simple and coherent use of the library in a distributed environment, the most relevant element is the definition of effective and flexible interfaces, that permit the development of efficient image processing applications. It can be achieved through a natural evolution from the library legacy code to the $\operatorname{PIMA}(\mathrm{GE})^{2}$ server; this step will directly provide a model to derive interfaces with the mentioned properties. The crucial element to achieve this goal becomes 
a classification and a hierarchical organization of the operations implemented by the library. Our idea is to group them in different objects and then outline a hierarchy of image processing operation objects. This effort is useful to provide the model for the definition of an adequate $\operatorname{PIMA}(\mathrm{GE})^{2}$ interface, in fact this structure will be codified using the CORBA Interface Description Language (IDL).

\subsection{The Legacy Code Classification}

During the classification of the library operations, we introduce conceptual objects in order to group together functions according to different rules. We consider one of distinguishing marks the similar nature or behavior of the operations; for example we grouped in the object Unary Operation the functions applied to individual pixels, i.e. square root, absolute value, etc. Another rule is the belonging to the same specialization field, for example in the object Differential Operations are grouped the differential operators, i.e. Gradient, Laplacian, Hessian; or we group together functions with the same data structures in input, for example functions changing the image geometry, i.e. rotation, translation and scaling, are grouped in the object Geometric Operations.

The result of the classification step is a set of image processing conceptual objects, made up of eight elements. They are not intended to prescribe how an operation is performed but to underline the operation similarity and to help in the definition of an effective and efficient interface. The classification does not put constraints on the code implementation and does not imply any code modification, because it is a conceptual step aimed to obtain an object oriented structure of the library code.

\subsection{The Hierarchical Library Organization}

Figure 1 represents the hierarchial library structure. The bottom layer of the hierarchy is represented by the main data structures of the library, that is the structures used to store images, convolution kernels, geometric matrices. We relate to them functions for memory management, I/O operations, and generic operations when it is possible, for example to transpose or invert a MATRIX. In this way we obtain three objects representing the basic elements of the server; they already include, as methods, some of the objects derived by the library classification step.

The second level of the $\operatorname{PIMA}(\mathrm{GE})^{2}$ server is a set of five objects, obtained by grouping together the library objects, according to the rules already mentioned. In this level we have Point Operations that take in input only one IMAGE object; Image Arithmetic Operations that take in input two IMAGE objects; Geometric Operations that take in input one IMAGE object and one MATRIX object; Convolution Operations that take in input one IMAGE object and one KERNEL object; Differential Operations that take in input one IMAGE and perform differential operators.

The top layer of the server is represented by the object Operations, it contains the image processing operations performed by the server and groups 
together the objects already described. Figure 1 explicitly visualizes the developed hierarchy and the inter-dependences among objects. The objects allow an easier management of the library operations, since a client is not more involved with a large number of functions, but he has to consider and to handle a small set of clear and well-defined objects.

\subsection{The PIMA(GE) $)^{2}$ Server Interface}

Since the hierarchy definition is completed, the $\operatorname{PIMA}(\mathrm{GE})^{2}$ interface implementing phase is totally planned and easily obtained. In fact the aim of the object hierarchy is to drive the definition of an adequate CORBA interface, and its IDL based implementation. Such interface represents the PIMA $(\mathrm{GE})^{2}$ API that will be called by Client applications. In this way the server provides a sequential API that hides the different levels and the computational complexity: underlying heterogeneous architectures, optimization policies, and parallel programming level. From the client point of view, the interface looks like a standard CORBA server, in fact the parallelism is totally hidden to the client, as well as the mechanisms and policies applied to allow MPI-CORBA compatibility, described in the next Sect.

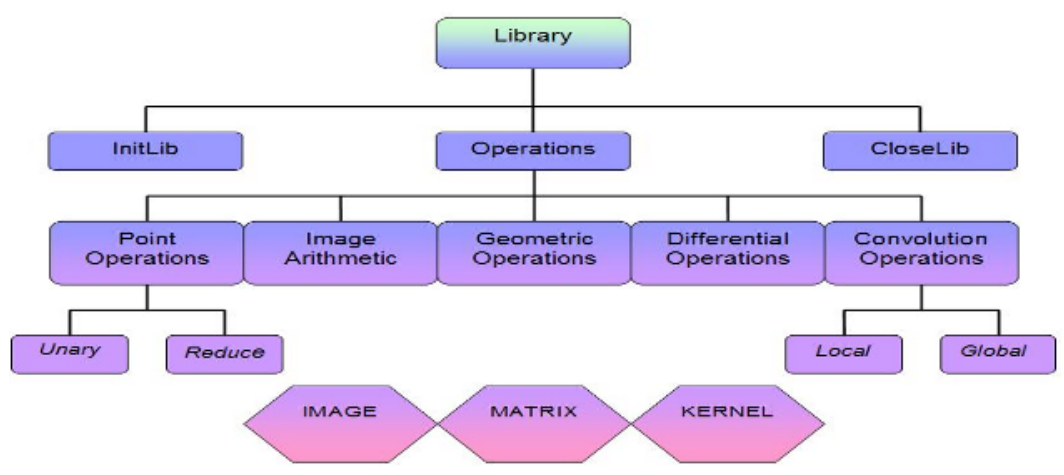

Fig. 1. Overview of the PIMA $(\mathrm{GE})^{2}$ hierarchy

\section{The Server Architecture}

The main difficulty in the $\operatorname{PIMA}(\mathrm{GE})^{2}$ implementation rises from the presence of parallelism in the computation. It imposes the management of two different environments: the CORBA framework (server side) and the MPI library (legacy code side). They are aimed to manage different kind of problems, therefore there is not a standard schema to allow their cooperation. In fact CORBA was born to develop mainly sequential applications, hence it does not support intrinsic compatibility with any kind of parallel environment. The use of CORBA to wrap parallel applications has been considered in different works, through the 
definition of parallel CORBA objects, [6, 16], or component environments built on CORBA [7, 5]. They all require the modification of CORBA middleware and hence are not standard solutions.

We decided to use a standard CORBA implementation, TAO [21, and look for a way allowing the development of parallel computations inside CORBA object. In order to achieve this goal we designed a special process to perform specific tasks. This process acts as the gateway that coordinates the two environments, allowing the computation. It has a dual position in the software architecture, in fact it will be in the same time a CORBA and a MPI process. On the CORBA side, it has to activate the ORB and perform as a CORBA server; at the same time it is also one of the spawned MPI processes. Acting as a MPI process, it will manage the parallel computation. This solution is possible because, acting as server CORBA, the designated process knows the client requirements an $\mathrm{d}$ can communicate them to the others MPI processes, can coordinate the computation and give back the informations to the clients. This solution does not require modifications to the legacy code, since it exploits the presence, in the legacy code, of the coordinator MPI process during the initialization and $\mathrm{I} / \mathrm{O}$ phases. The behavior is explained in the Fig. 2.

\section{Experimenting the Approach and Conclusions}

The experimental results we obtained show a reasonable overhead due to the presence of the CORBA wrapping framework. We considered an edge detection algorithm that calls different parallel library functions, and carried out three tests:

1. The parallel library is used in a normal MPI environment (i.e. without $\left.\operatorname{PIMA}(\mathrm{GE})^{2}\right)$;

2. $\operatorname{PIMA}(\mathrm{GE})^{2}$ is extended with the new algorithm and a remote client calls the new function;

3. A remote client calls the sequence of functions of $\operatorname{PIMA}(\mathrm{GE})^{2}$ that implements the algorithm.

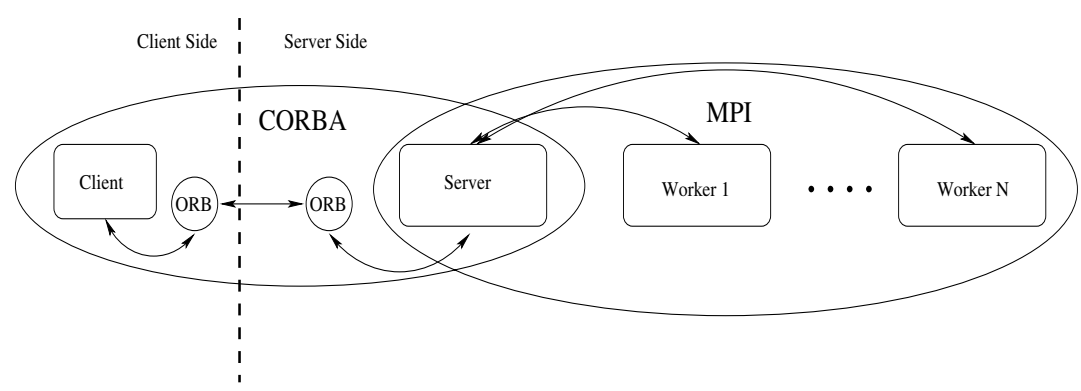

Fig. 2. The CORBA-MPI interaction 
Table 1. Experimental results, times are given in seconds

\begin{tabular}{lcccccc}
\hline Processes & 1 & 2 & 3 & 4 & 6 & 8 \\
\hline C++ MPI library & 1037.624 & 564.744 & 407.585 & 326.135 & 243.534 & 208.834 \\
PIMA(GE) ${ }^{2}$ one call & 1038.668 & 566.866 & 409.499 & 328.496 & 244.700 & 209.923 \\
PIMA $(\mathrm{GE})^{2}$ sequence & 1039.800 & 568.914 & 411.098 & 332.489 & 248.363 & 212.012 \\
\hline
\end{tabular}

Some general considerations apply to the three tests. In all the cases the parallel execution environment is a Linux cluster with eight nodes interconnected by a Gigabit switched Ethernet, and each node is equipped with a $2.66 \mathrm{GHz}$ Pentium processor, 512 Mbytes of RAM and two EIDE disks interface in RAID 0 . The remote client is a PC that does not belong to the cluster but is connected on the same LAN with a 10/100 Ethernet link. We avoid to use a "true" remote client, since we would like to measure the overhead due to $\operatorname{PIMA}(\mathrm{GE})^{2}$ infrastructure, without the possible unpredictable behaviour of an actual remote interconnection.

The same optimisation policy is used within the library in the three cases, in order to minimize the set-up operations, to optimise data distribution and to reduce data movement. Finally no actual images, but only symbolic identifiers are transmitted along the ORB. This decision is motivated by the fact that large size data transfer between the client and the server can be more efficiently accomplished in other way, like using optimised FTP protocols.

The three tests permit us to assess the performance quality of PIMA(GE) ${ }^{2}$. Table 1 reports execution times in second for the edge detection algorithm on 1 MByte image in the three cases and for different number of processors. The first test is necessary in order to assess the performance of the library and to define a term of comparison for PIMA(GE) ${ }^{2}$. With the second test we measured the overhead due to the CORBA set-up. As expected this set-up is a fixed time (about 2-3 seconds in our experiments) that does not depend on the number of parallel processes. In this case the interaction between the client and the server is very coarse grained and we have a minimum number of communications on the ORB. The third test is aimed to measure the performance degradation due to a more consistent use of the ORB communication channel. In fact in front of a single call of experiment 2, we have in this case a number of about 2166 calls and each one corresponds to a double communications on the ORB. Also in this case the overhead is quite limited especially for compute intensive applications. In this paper we have reported our experience in the design of PIMA $(\mathrm{GE})^{2}$ server interface. Future developments will be aimed to the integration of this CORBA based implementation with other Grid middleware.

\section{Acknowledgments}

This work has been supported by MIUR program L. 449/97-00 "High Performance Distributed Platform", by FIRB strategic project Grid.it, and by CNR strategic project @SWING. 


\section{References}

[1] Barros Jr, E.M., Shen, M., On Wangenheim, A.: A model for distributed medical image processing using CORBA. IEEE CNBS (2001) 189-

[2] The Cactus Project home page, www.cactuscode.org/

[3] The CORBA home page, http://www.corba.org/

[4] De Alfonso, C., Blanquer, I., Herniández, V.: Providing with High Performance 3D Medical Image Processing on a Distributed environment. Health GRID (2003)

[5] Dennis, A., Peréz C., Priol, T.: PadicoTM: An open integration framework for communication middleware and runtimes. IEEE Intl. CCGrid (2002) 144-151

[6] Dennis, A., Peréz C., Priol, T.: Achieving Portable and Efficient Parallel CORBA Objects. Concurrency and Computation: Practice and Experience, 1510 (2003) 891-909

[7] Dennis, A., Peréz C., Ribes, A.: Padico: a component-based software infrastructure for Grid Computing. IPDPS (2003) 2

[8] Forster, I., Kesselman, C.: The grid: blueprint for a new computing infrastructure. 2nd Edition Morgan Kaufmann (2004)

[9] Galizia, A.: Evaluation of optimization policies in the impletation of Parallel Libraries. Technical Report IMATI-CNR-Ge 20 (2004)

[10] GrADS Project Home Page, http://www.hipersoft.rice.edu/grads/

[11] Hastings, S., Kurc, T., Langella, S., Catalyurek, U., Pan T., Saltz, J.: Image Processing for the Grid: a toolkit for building Grid-enable Image Processing Applications. IEEE/AMc CCGRID (2003) 36-43

[12] Lebak, J., Kepner, J., Hoffmann, H., Rudtledge, E.: Parallel VSIPL++: an open standard library for high-performance parallel signal processing. IEEE Proceedings 932 February (2005) 313-330

[13] MPI Home Page, http://www-unix.mcs.anl.gov/mpi/

[14] NetSolve Home Page, http://icl.cs.utk.edu/netsolve/

[15] OMG Official Website, http://omg.org

[16] Peréz, C., Priol, T., Ribes, A.: A Parallel CORBA Component Model. INRIA Technical Report N.4552 September (2002)

[17] PIPT Home Page, http://www.osl.iu.edu/research/pipt

[18] Ritter, G., Wilson, J.: Handbook of Computer Vision Algorithms in Image Algebra. 2nd edition CRC Press Inc (2001)

[19] Seinstra, F., Koelma, D., Geusebroek, J.M.: A software architecture for user transparent parallel image processing. Parallel Computing 28 7-8 (2002) 967-993

[20] Sneed, H.M.: Encapsulation of Legacy Software: A technique for reuse software components. Annals of Software Engineering 9 (2000) 293-313

[21] Tao home page, http://www.cs.wustl.edu/ ${ }^{\sim}$ schmidt/TAO.html 